\title{
Acute Disseminated Encephalomyelitis Following Immunization with Human Papillomavirus Vaccines
}

\author{
Makoto Yoneda \\ Key words: HPV vaccines, ADEM, adverse effects, adjuvants, epidemiologic study
}

(Intern Med 55: 3077-3078, 2016)

(DOI: 10.2169/internalmedicine.55.7217)

The immunization of adolescent girls with the human papilloma virus (HPV) vaccines, a bivalent Cervarix ${ }^{\circledR}$ and a quadrivalent Gardasil $^{\circledR}$, has been widely introduced to prevent uterine and cervical cancer (1). Since the regulatory approval of the first quadrivalent HPV vaccine in 2006 and later the bivalent HPV vaccine, as of 2011, about 120 million doses have been distributed worldwide (1). Since 2010, both vaccines have been introduced for administration to adolescent girls in Japan. However, with the rise in the number of subjects receiving this vaccine, some adverse effects have also started to be reported in Japan. These adverse effects comprise varied clinical manifestations, such as orthostatic intolerance; postural tachycardia syndrome; pain or coldness of ambiguous origin in the limbs, as with complex regional pain syndrome; involuntary movements; abnormal behavior resembling psychosis; headaches; general fatigue; Guillain-Barré syndrome; acute cerebellar ataxia; and acute disseminated encephalomyelitis (ADEM) (2-6).

Sekiguchi et al. reported two cases of ADEM after the administration of HPV vaccines in this issue (7). Both patients presented with neurological symptoms after the second administration of the HPV vaccine. Their symptoms improved after the intravenous administration of methylprednisolone, with no sign of relapse. The authors estimated a prevalence of 0.05 per 100,000 for ADEM after the HPV vaccine administration in Japan, according to the data from the Pharmaceutical and Medical Devices Agency (PMDA; c.f. 0.10.2 per 100,000 of post-vaccination ADEM in total) (8), and emphasized the importance of further data accumulation in their survey of the relationship between the HPV vaccines and ADEM (7). In a large epidemiologic study, ADEM preceded by vaccination was infrequent but not rare (around $5 \%$ ) (9) and tended to occur after the booster vaccination rather than after the first application, just as in the cases reported in this issue $(6,7)$.

In general, immunization via vaccines can induce experi- mental allergic encephalitis (EAE), probably due to the molecular mimicry between the vaccine epitope and neural antigens with the subsequent activation of cross-reactive immune cells $(10,11)$. Alternatively, autoimmune/inflammatory syndrome induced by adjuvants (ASIA) has also been proposed to be a potential pathophysiology of the adverse effects noted with HPV vaccines (12). In particular, aluminum (Al) is commonly used in the HPV vaccines as an adjuvant to prolong the duration of the vaccine effects. Indeed, behavioral abnormalities have been observed in young female mice following administration of aluminum adjuvants with the HPV vaccine (13).

These experimental data support the relationship between neurological manifestations and HPV vaccines. However, conflicting findings have also been reported. For example, a large cohort study in Denmark and Sweden exploring the relationship between the HPV vaccines and potential adverse effects failed to demonstrate this association statistically (14). Although the adverse effects, including ADEM, after the HPV-vaccination epidemiologically remain obscure, clinicians should continuously pay attention to the possibility of adverse effects of HPV vaccines in daily medical practice. ADEM after HPV vaccination is distinct but closely related to the adverse effects of HPV vaccination, which comprises a broad spectrum of neurological symptoms with no evidence beyond obvious laboratory and radiological abnormalities at this time. A nationwide survey examining the link between the clinical manifestations and HPV vaccines including a statistical analysis and the establishment of targeted treatments in Japan is urgently needed, as well as experimental research to clarify the pathophysiology.

The author states that he has no Conflict of Interest (COI). 


\section{References}

1. FUTURE II Study Group. Quadrivalent vaccine against human papillomavirus to prevent high-grade cervical lesions. N Engl J Med 356: 1915-1927, 2007.

2. Brinth LS, Pors K, Theibel AC, Mehlsen J. Orthostatic intolerance and postural tachycardia syndrome as suspected adverse effects of vaccination against human papilloma virus. Vaccine 33: 26022605, 2015.

3. Kinoshita T, Ikeda S. Peripheral sympathetic nerve dysfunction in adolescent Japanese girls following immunization with the human papillomavirus vaccine. Intern Med 53: 2185-2200, 2014.

4. Souayah N, Michas-Martin PA, Nasar A, et al. Guillain-Barré syndrome after Gardasil vaccination: data from Vaccine Adverse Event Reporting System 2006-2009. Vaccine 29: 886-889, 2011.

5. Yonee C, Toyoshima M, Maegaki Y, et al. Association of acute cerebellar ataxia and human papilloma virus vaccination: a case report. Neuropediatrics 44: 265-267, 2013.

6. Wildemann B, Jarius S, Hartmann M, Regula JU, Hametner C. Acute disseminated encephalomyelitis following vaccination against human papilloma virus. Neurology 72: 2132-2133, 2009.

7. Sekiguchi K, Yasui N, Kowa H, Kanda F, Toda T. Two cases of acute disseminated encephalomyelitis following vaccination against human papilloma virus. Intern Med 55: 3181-3184, 2016.

8. Menge T, Kieseier BC, Nessler S, Hemmer B, Hartung HP, Stüve O. Acute disseminated encephalomyelitis: an acute hit against the brain. Curr Opin Neurol 20: 247-254, 2007.

9. Leake JA, Albani S, Kao AS, et al. Acute disseminated encephalomyelitis in childhood: epidemiologic, clinical and laboratory features. Pediatr Infect Dis J 23: 756-764, 2004.

10. Baxter AG. The origin and application of experimental autoimmune encephalomyelitis. Nat Rev Immunol 7: 904-912, 2007.

11. Tenembaum S, Chitnis T, Ness J, Hahn JS; International Pediatric MS Study Group. Acute disseminated encephalomyelitis. Neurology 68 (16 Suppl 2): S23-S36, 2007.

12. Shoenfeld Y, Agmon-Levin N. 'ASIA' - autoimmune/inflammatory syndrome induced by adjuvants. J Autoimmun 36: 4-8, 2011.

13. Inbar R, Weiss R, Tomljenovic L, et al. WITHDRAWN: Behavioral abnormalities in young female mice following administration of aluminum adjuvants and the human papillomavirus (HPV) vaccine Gardasil. Vaccine (in press).

14. Arnheim-Dahlströ L, Pasternak B, Svanström H, Sparén P, Hviid A. Autoimmune, neurological, and venous thromboembolic adverse events after immunization of adolescent girls with quadrivalent human papillomavirus vaccine in Denmark and Sweden: cohort study. BMJ 347: f5906, 2013.

The Internal Medicine is an Open Access article distributed under the Creative Commons Attribution-NonCommercial-NoDerivatives 4.0 International License. To view the details of this license, please visit (https://creativecommons.org/licenses/ by-nc-nd/4.0/).

(C) 2016 The Japanese Society of Internal Medicine http://www.naika.or.jp/imonline/index.html 